\title{
Post Hoc Analysis of the CONFIDENCE II, PROTECT I, SHAKE THE HABIT I and SHAKE THE HABIT II Observational Studies in Mild to Moderate Hypertensive Patients Treated with Perindopril and Atorvastatin Concomitantly
}

\author{
John S. Sampalis ${ }^{1,2} \cdot$ Eliofotisti Psaradellis $^{1} \cdot$ Melissa Stutz $^{1} \cdot$ Jenaya Rickard $^{1} \cdot$ Emmanouil Rampakakis $^{1}$
}

Published online: 17 November 2018

(c) The Author(s) 2018

\begin{abstract}
Background and Objectives Management of hypertension and dyslipidemia is important when considering cardiovascular disease risk; however, achievement of optimal lipid and blood pressure (BP) targets in clinical practice remains inadequate. This analysis sought to estimate the frequency, effectiveness, and safety of co-administrated atorvastatin and perindopril in routine care.

Methods We conducted a post hoc analysis of four Canadian, prospective, multi-center, observational studies assessing real-life effectiveness and safety of perindopril + atorvastatin in mild-to-moderate hypertensive patients with concomitant dyslipidemia over 16 weeks. The safety population comprised patients receiving one or more doses of free combination perindopril + atorvastatin; the full analysis set (FAS) received perindopril + atorvastatin at baseline, with one or more postbaseline systolic BP measurements while on treatment.

Results A total of 3541 and 3172 patients were included in the safety population and FAS, respectively. At the last observation carried forward, significant reductions in mean systolic BP $(-18.0 \mathrm{mmHg} ; p<0.001)$ and diastolic $\mathrm{BP}(-8.9 \mathrm{mmHg}$; $p<0.001$ ) were observed; target BP was achieved by $73.1 \%$ of patients. Emergent adverse events (AEs) were reported in $8.0 \%$ of patients, the most common being cough ( $4.5 \%$ of patients), headache $(0.9 \%)$, and dizziness $(0.8 \%)$. Four serious AEs were reported among three $(0.1 \%)$ patients. No differences were observed in effectiveness or safety between studies.

Conclusions Concomitant perindopril + atorvastatin therapy demonstrated similar efficacy across all studies, with significant reductions in BP and achievement of target BP levels observed in a real-world setting. Results align with known safety profiles of atorvastatin and perindopril, with no unexpected AEs observed when compared with data from treatment with the individual drugs.
\end{abstract}

John S. Sampalis

jsampalis@jssresearch.com

$\triangle$ Emmanouil Rampakakis

submissions@jssresearch.com

Eliofotisti Psaradellis

epsaradellis@jssresearch.com

Melissa Stutz

mstutz@jssresearch.com

Jenaya Rickard

jrickard@jssresearch.com

1 JSS Medical Research, 9400 boul. Henri-Bourassa Ouest, St-Laurent, QC H4S 1N8, Canada

2 McGill University, Montreal, QC, Canada

\section{Key Points}

Post hoc results find perindopril + atorvastatin co-administration safe and effective for the treatment of patients with mild-to-moderate hypertension and concomitant dyslipidemia in a real-world setting.

Results support perindopril + atorvastatin free drug combination regimens. 


\section{Introduction}

Hypertension, or high blood pressure (BP), is a universal concern that was designated the leading risk factor for global disease burden in 2010 [1]. Hypertension is often labeled a 'silent killer' due to the fact that early symptoms are rare to non-existent, and it is a major risk factor for cardiovascular disease (CVD), including congestive heart failure (CHF), aneurysm, and stroke [2]. Dyslipidemia, another major risk factor for CVD, frequently occurs in hypertensive patients, concurrently affecting between $15 \%$ and $31 \%$ of hypertensive patients [3]. Moreover, concomitant dyslipidemia and hypertension compound the risk for CVD, more so than the sum of the risks associated with the individual disorders $[4,5]$.

The management of both hypertension and dyslipidemia is important when considering CVD risk; however, there is evidence suggesting that hypertensive patients with comorbid dyslipidemia are often not treated with a lipid-lowering agent, or are receiving treatment at a sub-optimal dose [6]. Despite the known importance of controlling modifiable risk factors of CV disease, achievement of optimal lipid [6-8] and BP [9-13] targets remains inadequate. Specifically, with respect to hypertension, a recent report from the American Heart Association found that only slightly more than half (54.1\%) of patients were considered to have controlled hypertension [13]. Furthermore, adherence appears to be a major obstacle resulting in a variety of adverse outcomes among patients taking cardio-protective medications, including angiotensin-converting enzyme inhibitors (ACEIs) and statins [14].

The objectives of this analysis were to identify the frequency of concomitant perindopril and atorvastatin administration in mild to moderate hypertensive patients who were followed in routine care and to assess the real-life effectiveness and safety of perindopril when taken in combination with atorvastatin.

\section{Methods}

\subsection{Study Design}

This was a post hoc analysis of the following four Canadian, prospective cohort, multi-center, observational studies, described in Table 1, which involved mild to moderate hypertensive patients, 18 years of age and older, either uncontrolled or not previously treated [15-18]. A total of 51,821 patients were enrolled across studies, of which 43,444 received at least one dose of perindopril (intentto-treat [ITT] population). Patients were followed for 16 $( \pm 2)$ weeks, with two recommended follow-up visits at 4
$( \pm 2)$ and $16( \pm 2)$ weeks after their first visit/inclusion in the study. Considering that all four studies shared that same inclusion/non-inclusion criteria and duration of perindopril therapy, a post hoc analysis was conducted to identify the sub-population of patients in the four studies who were treated concomitantly with perindopril and atorvastatin, and to assess the effectiveness and safety of this free combination. The decision to treat patients with perindopril was independent of study participation and decided upon by the treating physicians prior to screening/enrolment in the four studies. After the physician's decision to prescribe perindopril, a voluntary informed consent form was obtained from the patient in order to initiate participation into the study. Assessment of the inclusion and exclusion criteria was based on the patient's medical history and the treating physician's assessment and judgment.

\subsection{Post Hoc Study Population}

The study population included four groups of adult ( $\geq 18$ years) patients with mild to moderate uncontrolled hypertension $(140 \leq$ systolic blood pressure $[\mathrm{SBP}] \leq 179 \mathrm{mmHg}$ and/or $90 \leq$ diastolic blood pressure $[\mathrm{DBP}] \leq 109 \mathrm{mmHg}$; for diabetic patients, $130 \leq \mathrm{SBP} \leq 179 \mathrm{mmHg}$ and/or $80 \leq \mathrm{DBP} \leq 109 \mathrm{mmHg}$ ), either uncontrolled with no previous perindopril treatment or not previously treated, without unstable coronary artery disease or recent myocardial infarction, who had concomitant administration of perindopril and atorvastatin at baseline and at least one post-baseline assessment, no fixed-dose combination pill, between (i) 2008 and 2010 in CONFIDENCE II, (ii) 2009 and 2011 in PROTECT I, (iii) 2010 and 2012 in Shake the Habit (STH) I, and (iv) 2012 and 2014 in STH II. Atorvastatin may have been initiated prior to perindopril or at baseline.

\subsection{Outcome Measures}

The outcome measures used to evaluate perindopril effectiveness when taken concomitantly with atorvastatin were the change from baseline (Visit 1) for SBP and DBP at Visit 2, Visit 3, and LOCF (last observation carried forward), and percentage of patients who achieved target BP as per the most recent (2013) joint European Society of Hypertension/European Society of Cardiology (ESH/ESC) hypertension guidelines available at the time of analysis ( $<140 / 90 \mathrm{mmHg}$, or $<140 / 85 \mathrm{mmHg}$ for diabetic patients) [19]. Individual study protocols defined target BP as per the 2012 Canadian Hypertension Education Program (CHEP) guidelines, which differed from the European guidelines with respect to the definition of target BP for diabetic patients $(<130 / 80 \mathrm{mmHg})$ [20]. Considering that the perprotocol definition, and resultant inclusion criterion, defined 


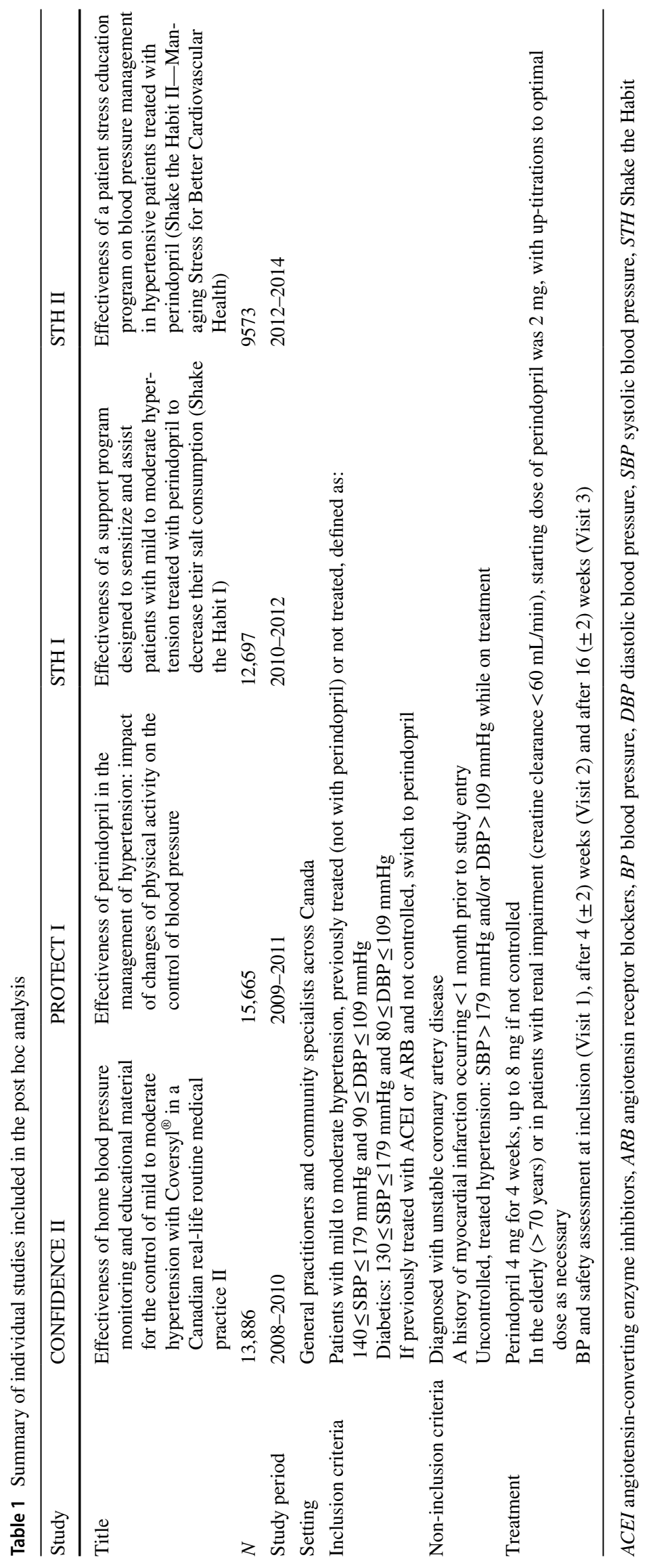


uncontrolled hypertension in diabetics according to the CHEP guidelines, analyses of target BP achievement did not include diabetic patients, who, at baseline, were considered controlled due to post hoc adoption of the ESH/ESC definition.

During all four studies, BP measures were evaluated in-office, at baseline and during each of the three study visits; both SBP and DBP were measured with the patient in a seated position, having been seated for at least $5 \mathrm{~min}$, according to a standardized BP measurement protocol, which was provided to all study sites. The average of two BP measurements, taken 5 min apart, was used to assess BP control. Although CONFIDENCE II patients may have also measured BP at home, only in-office BP readings were considered in the current post hoc analysis.

The safety of concomitant perindopril and atorvastatin was assessed by reports of emergent adverse events (EAEs), and emergent serious adverse events (ESAEs), which were coded using MedDRA version 17.0. Safety was ascertained as per the physician's judgment at Visits 2 and 3.

\subsection{Statistical Methods}

All statistical analyses were performed for all patients during the treatment period (concomitant perindopril and atorvastatin) only, and stratified by the individual studies. Descriptive statistics, including the mean and standard deviation (SD) for continuous variables, and frequency distributions for categorical variables, were produced for patient demographics and baseline characteristics on the full analysis set (FAS), comprised of all patients receiving concomitant perindopril and atorvastatin at baseline who had at least one followup SBP measurement while on free combination treatment. Effectiveness analyses were performed on the FAS and assessed descriptively, as described above. Absolute changes in SBP and DBP to Visit 3 were conducted applying the LOCF method, with missing SBP or DBP data at Visit 3 imputed from Visit 2 values. Within-group improvements in SBP and DBP to LOCF were assessed with the One-Sample $T$ test. Safety analyses were performed on the safety set, which included all patients receiving concomitant perindopril and atorvastatin at least one time during follow-up, and were evaluated descriptively using frequency distributions.

\section{Results}

\subsection{Patient Disposition}

Of the 43,444 patients in the ITT population, 3541 and 3172 patients were included in the safety population and the FAS, respectively (Fig. 1).

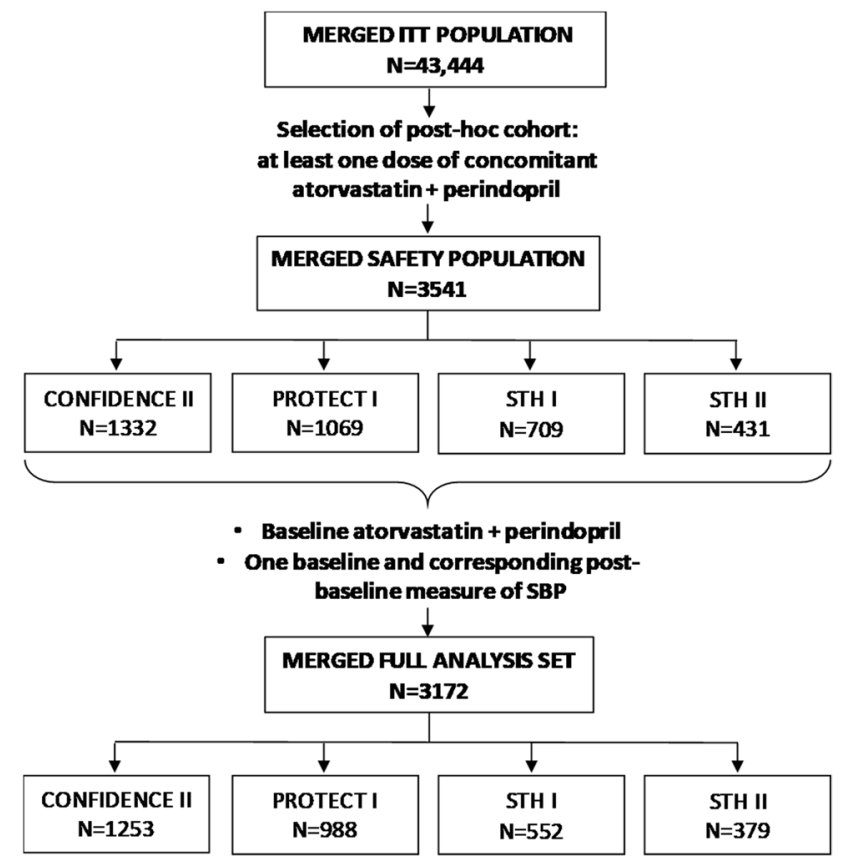

Fig. 1 Patient flow chart. The merged ITT population included all patients with a baseline assessment that took at least one dose of the study medication (perindopril) and had a baseline SBP measurement. From this merged ITT, the study populations for the post hoc analyses included (i) all those who received at least one dose of combination perindopril + atorvastatin at any point during the study (merged safety population), (ii) all those patients who received combination perindopril + atorvastatin at baseline, and for whom a baseline and one or more follow-up SBP assessment were available (merged full analysis set). ITT intent-to-treat, SBP systolic blood pressure, $S T H$ Shake the Habit

Among the FAS, 81 (2.5\%) patients withdrew from the study, 309 (9.7\%) patients were lost to follow up, and 2782 (87.7\%) patients completed the study (Table 2 ).

\subsection{Baseline Characteristics}

The majority of patients were male, with mean (SD) age ranging from 64.7 (11.5) to 65.7 (11.5) years in PROTECT II and STH I, respectively, and with BMI (mean [SD]) lowest in CONFIDENCE I $\left(29.3[5.7] \mathrm{kg} / \mathrm{m}^{2}\right)$ and highest in STH II $\left(29.8[6.4] \mathrm{kg} / \mathrm{m}^{2}\right)$. Baseline SBP was approximately $150 \mathrm{mmHg}$ for patients in CONFIDENCE II, STH I, and STH II, and was slightly lower (mean [SD]: 147.4 [10.1] $\mathrm{mmHg}$ ) for PROTECT I patients. Mean (SD) DBP ranged from 86.0 (9.3) $\mathrm{mmHg}$ to $87.3(8.4) \mathrm{mmHg}$ across studies (Table 3).

Across all studies, rates of baseline cardiovascular risk factors were high: physical inactivity was reported by nearly two-thirds of patients in CONFIDENCE II (61.5\%; $n=771 / 1253$ ), and in approximately half of patients in PROTECT I (46.9\%; $n=463 / 988)$, STH I $(49.5 \% ; n=273 / 552)$, 
Table 2 Patient disposition by study and for the merged full analysis set

\begin{tabular}{lccccc}
\hline Status & $\begin{array}{l}\text { CONFIDENCE II } \\
(N=1253)\end{array}$ & $\begin{array}{l}\text { PROTECT I } \\
(N=988)\end{array}$ & $\begin{array}{l}\text { STH I } \\
(N=552)\end{array}$ & $\begin{array}{l}\text { STH II } \\
(N=379)\end{array}$ & $\begin{array}{c}\text { Merged FAS } \\
(N=3172)\end{array}$ \\
\hline Withdrawn, $n(\%)$ & $35(2.8)$ & $24(2.4)$ & $0(0.0)$ & $22(5.8)$ & $81(2.5)$ \\
Withdrew consent & $5(0.4)$ & $1(0.1)$ & $0(0.0)$ & $1(0.3)$ & $7(0.2)$ \\
Adverse event & $23(1.8)$ & $17(1.7)$ & $0(0.0)$ & $17(4.5)$ & $57(1.8)$ \\
Lack of drug efficacy & $1(0.1)$ & $0(0.0)$ & $0(0.0)$ & $0(0.0)$ & $1(0.03)$ \\
Protocol violation & $1(0.1)$ & $0(0.0)$ & $0(0.0)$ & $0(0.0)$ & $1(0.03)$ \\
Non-compliance & $1(0.1)$ & $2(0.2)$ & $0(0.0)$ & $1(0.3)$ & $4(0.1)$ \\
Other & $4(0.3)$ & $4(0.4)$ & $0(0.0)$ & $3(0.8)$ & $11(0.3)$ \\
Lost to follow up, $n(\%)$ & $121(9.7)$ & $65(6.6)$ & $78(14.1)$ & $45(11.8)$ & $309(9.7)$ \\
Completed, $n(\%)$ & $1097(87.5)$ & $899(91.0)$ & $474(85.9)$ & $312(82.3)$ & $2782(87.7)$ \\
\hline
\end{tabular}

FAS full analysis set, $S T H$ Shake the Habit

Table 3 Baseline demographic and patient characteristics by study and for the merged full analysis set

\begin{tabular}{|c|c|c|c|c|c|}
\hline & $\begin{array}{l}\text { CONFIDENCE II } \\
(N=1253)\end{array}$ & $\begin{array}{l}\text { PROTECT I } \\
(N=988)\end{array}$ & $\begin{array}{l}\text { STH I } \\
(N=552)\end{array}$ & $\begin{array}{l}\text { STH II } \\
(N=379)\end{array}$ & $\begin{array}{l}\text { Merged FAS } \\
(N=3172)\end{array}$ \\
\hline Age, years, mean (SD) & $64.9(11.3)$ & $64.7(11.5)$ & $65.4(11.7)$ & $65.7(11.5)$ & $65.0(64.6)$ \\
\hline BMI, kg/m², mean (SD) & $29.3(5.7)$ & $29.4(5.4)$ & $29.5(6.4)$ & $29.8(6.4)$ & $29.4(5.8)$ \\
\hline Gender, male, $n(\%)$ & $704(56.2)$ & $582(58.9)$ & $346(62.7)$ & $199(52.5)$ & $1831(57.7)$ \\
\hline SBP, mmHg, mean (SD) & $150.2(11.6)$ & $147.4(10.1)$ & $150.0(9.7)$ & $150.7(9.6)$ & $149.4(10.6)$ \\
\hline DBP, mmHg, mean (SD) & $86.7(8.8)$ & $85.7(8.5)$ & $87.3(8.4)$ & $86.0(9.3)$ & $86.4(8.7)$ \\
\hline \multicolumn{6}{|l|}{ Cardiovascular risk factors, yes, $n(\%)$} \\
\hline Physical inactivity & $771(61.5)$ & $463(46.9)$ & $273(49.5)$ & $214(56.5)$ & $1721(54.3)$ \\
\hline Current smoker ${ }^{\mathrm{a}}$ & $241(19.2)$ & $170(17.2)$ & $98(17.8)$ & $64(16.9)$ & $573(18.1)$ \\
\hline Obesity $^{\mathrm{b}}$ & $495(39.5)$ & 355 (35.9) & $211(41.4)$ & $152(40.1)$ & $1213(38.8)$ \\
\hline Cholesterol ratio $^{c}$ & $348(27.8)$ & $293(29.7)$ & $200(36.2)$ & $109(28.8)$ & $950(29.9)$ \\
\hline Coronary artery disease & $370(29.5)$ & $243(24.6)$ & $212(38.4)$ & $123(32.5)$ & $948(29.9)$ \\
\hline Diabetes (I/II) & $572(45.7)$ & $480(48.6)$ & $258(46.7)$ & $184(48.5)$ & 1447 (45.6) \\
\hline Microalbuminuria & $197(15.7)$ & $64(6.5)$ & $61(11.1)$ & $60(15.8)$ & $382(12.0)$ \\
\hline Baseline concomitant CV medication, yes, $n(\%)$ & $952(76.0)$ & 703 (71.2) & $370(67.0)$ & $248(65.4)$ & $2273(71.7)$ \\
\hline Calcium channel blockers & 337 (26.9) & $196(19.8)$ & $86(15.6)$ & $77(20.3)$ & $696(21.9)$ \\
\hline Beta blockers & $351(28.0)$ & $256(25.9)$ & $157(28.4)$ & $105(27.7)$ & $869(27.4)$ \\
\hline Diuretics & $302(24.1)$ & $231(23.4)$ & $67(12.1)$ & $61(16.1)$ & $661(20.8)$ \\
\hline ASA/antiplatelets & $768(61.3)$ & $541(54.9)$ & $240(43.5)$ & $111(29.3)$ & $1160(52.3)$ \\
\hline Cardiac therapy & $780(62.3)$ & $546(55.3)$ & $247(44.7)$ & $119(31.4)$ & $1692(53.3)$ \\
\hline Antihypertensive therapy & $652(52.0)$ & $494(50.0)$ & $251(45.5)$ & $211(55.7)$ & $1608(50.7)$ \\
\hline \multicolumn{6}{|l|}{$\begin{array}{l}\text { Change from baseline in concomitant CV medica- } \\
\text { tion, yes, } n(\%)\end{array}$} \\
\hline Visit $2^{\mathrm{d}}$ & $56(4.5)$ & $28(2.8)$ & $18(3.3)$ & $10(2.6)$ & $112(3.5)$ \\
\hline Visit $3^{\mathrm{e}}$ & $61(5.6)$ & $73(8.1)$ & $18(3.8)$ & $18(5.4)$ & $170(6.1)$ \\
\hline
\end{tabular}

${ }^{a}$ Defined as more than one cigarette/day

${ }^{\mathrm{b}} \mathrm{BMI} \geq 30 \mathrm{~kg} / \mathrm{m}^{2}$

${ }^{\mathrm{c}}$ Cholesterol ratio $\geq 6$. Ratio determined as total cholesterol/HDL

${ }^{\mathrm{d}}$ Percentages based on total number of patients who completed Visit $2(n=3172)$

${ }^{\text {e}}$ Percentages based on total number of patients who completed Visit $3(n=2802)$

$A S A$ acetylsalicylic acid, $B M I$ body mass index, $C V$ cardiovascular, $D B P$ diastolic blood pressure, $F A S$ full analysis set, $H D L$ high-density lipoprotein, $S B P$ systolic blood pressure, $S D$ standard deviation, $S T H$ Shake the Habit 
and STH II (56.5\%; $n=214 / 379)$. Prevalence of diabetes mellitus was $>45 \%$ across all study populations, with obesity rates at approximately $36 \%$ in PROTECT I patients, and $40 \%$ in CONFIDENCE I, STH I, and STH II populations. Prevalence of a high cholesterol ratio (total cholesterol/ HDL $\geq 6$ ) and coronary artery disease ranged from $27.8 \%$ $(n=348 / 1253$; PROTECT I) to $36.2 \%(200 / 552 ;$ STH I), and from $24.6 \%(n=243 / 988$; PROTECT I) to $38.4 \%$ $(n=212 / 552 ;$ STH I), respectively. Current smokers (defined as more than one cigarette per day) constituted similar proportions of all four study populations, with this percentage being highest in the CONFIDENCE I study population $(n=241 / 1253 ; 19.2 \%)$ (Table 3$)$.

Concomitant cardiovascular (CV) medication at baseline was reported by between 65.4 and $76.0 \%$ of patients. ASA/ antiplatelet medication, cardiac therapy, and other antihypertensive therapies were the most predominately administered concomitant $\mathrm{CV}$ medications, reported by approximately half of all patients, each, in the merged FAS (Table 3). In individual studies, the proportion of patients prescribed ASA/antiplatelets and cardiac therapy was variable, ranging from approximately $30-60 \%$ of patients. The proportion of patients receiving concomitant antihypertensive therapy, however, was comparable across studies with $46-56 \%$. The change from baseline in $\mathrm{CV}$ medications remained relatively stable across all visits, with a maximum $4.5 \%$ (CONFIDENCE I; $n=56 / 1253$ ) and $8.1 \%$ (PROTECT I; $n=73 / 896$ ) of patients reporting a change in $\mathrm{CV}$ medication at Visits 2 and 3, respectively. Overall, in the merged FAS, a CV medication change was noted in $3.5 \%$ of patients at Visit 2 $(n=112 / 3172)$ and $6.1 \%$ of patients at Visit $3(n=170 / 2802)$ (Table 3).

\subsection{Atorvastatin/Perindopril Dosing}

At Visit 1, the most frequent dose of perindopril was the $4 \mathrm{mg} /$ day across all four studies. Atorvastatin dose at baseline was most frequently $10 \mathrm{mg} /$ day in CONFIDENCE II, PROTECT I, and STH II (range 27.4-34.8\%), and $40 \mathrm{mg}$ / day in STH I (31.5\%). In these studies, 41.7\% $(n=523 / 1253$; CONFIDENCE II), 32.4\% ( $n=320 / 988$; PROTECT I), $60.3 \%(n=333 / 552$; STH I), and 53.8\% $(n=204 / 379$; STH II) of patients remained on the same dose of perindopril + atorvastatin until the last visit (Table 4).

Table 4 Dose of perindopril + atorvastatin combination by study and for the merged full analysis set

\begin{tabular}{|c|c|c|c|c|c|}
\hline & $\begin{array}{l}\text { CONFIDENCE II } \\
(N=1253)\end{array}$ & $\begin{array}{l}\text { PROTECT I } \\
(N=988)\end{array}$ & $\begin{array}{l}\text { STH I } \\
(N=552)\end{array}$ & $\begin{array}{l}\text { STH II } \\
(N=379)\end{array}$ & $\begin{array}{l}\text { Merged FAS } \\
(N=3172)\end{array}$ \\
\hline \multicolumn{6}{|l|}{ Baseline perindopril dose, $n(\%)$} \\
\hline $2 \mathrm{mg}$ & $219(17.5)$ & $215(21.8)$ & $49(8.9)$ & $29(7.7)$ & $512(16.1)$ \\
\hline $4 \mathrm{mg}$ & $956(76.3)$ & $688(69.6)$ & $386(69.6)$ & $270(71.2)$ & $2300(72.5)$ \\
\hline $6 \mathrm{mg}$ & $0(0.0)$ & $0(0.0)$ & $0(0.0)$ & $1(0.3)$ & $1(0.03)$ \\
\hline $8 \mathrm{mg}$ & $62(4.9)$ & $58(5.9)$ & $114(20.7)$ & $75(19.8)$ & $309(9.7)$ \\
\hline $16 \mathrm{mg}$ & $0(0.0)$ & $1(0.1)$ & $0(0.0)$ & $0(0.0)$ & $1(0.03)$ \\
\hline Missing & $16(1.3)$ & $26(2.6)$ & $3(0.5)$ & $4(1.1)$ & $49(1.5)$ \\
\hline \multicolumn{6}{|l|}{ Baseline atorvastatin dose, $n(\%)$} \\
\hline $5 \mathrm{mg}$ & $2(0.2)$ & $3(0.3)$ & $0(0.0)$ & $6(1.6)$ & $11(0.3)$ \\
\hline $10 \mathrm{mg}$ & $343(27.4)$ & $339(34.3)$ & $147(26.6)$ & $132(34.8)$ & $961(30.3)$ \\
\hline $15 \mathrm{mg}$ & $1(0.1)$ & $1(0.1)$ & $0(0.0)$ & $1(0.3)$ & $3(0.1)$ \\
\hline $20 \mathrm{mg}$ & $339(27.1)$ & $233(23.6)$ & $170(30.8)$ & $119(31.4)$ & $861(27.1)$ \\
\hline $30 \mathrm{mg}$ & $4(0.3)$ & $1(0.1)$ & $3(0.5)$ & $4(1.1)$ & $12(0.4)$ \\
\hline $40 \mathrm{mg}$ & $176(14.0)$ & $112(11.3)$ & $174(31.5)$ & $86(22.7)$ & $548(17.3)$ \\
\hline$>40$ to $<80 \mathrm{mg}$ & $5(0.4)$ & $0(0.0)$ & $5(0.9)$ & $6(1.6)$ & $16(0.5)$ \\
\hline $80 \mathrm{mg}$ & $39(3.1)$ & $24(2.4)$ & $38(6.9)$ & $19(5.0)$ & $120(3.8)$ \\
\hline$>80 \mathrm{mg}$ & $0(0.0)$ & $0(0.0)$ & $3(0.5)$ & $0(0.0)$ & $3(0.1)$ \\
\hline Missing & $344(27.5)$ & $275(27.8)$ & $12(2.2)$ & $6(1.6)$ & $637(20.1)$ \\
\hline \multicolumn{6}{|l|}{ Dose of atorvastatin + perindopril, $n(\%)$} \\
\hline Remained on same dose until last available visit & $523(41.7)$ & $320(32.4)$ & $333(60.3)$ & $204(53.8)$ & $1380(43.5)$ \\
\hline Changed dose for at least one drug & $334(26.7)$ & $368(37.2)$ & $162(29.3)$ & $145(38.3)$ & $1009(31.8)$ \\
\hline At least one drug dose was missing & $396(31.6)$ & $300(30.4)$ & $57(10.3)$ & $30(7.9)$ & $783(24.7)$ \\
\hline
\end{tabular}

FAS full analysis set, STH Shake the Habit 
Fig. 2 SBP (a) and DBP (b) reduction in patients receiving perindopril + atorvastatin. Results are presented by visit. Absolute change (SD) in SBP and DBP indicated above respective bar graphs. Withingroup testing was performed for the LOCF; significant changes $(p<0.001$; denoted by ' $*$ ') in SBP and DBP from baseline were observed at the LOCF for blood pressure, $F A S$ full analysis set, $L O C F$ last observation carried forward, $S B P$ systolic blood pressure, $S D$ standard deviation, STH Shake the Habit, $\Delta$ change all comparisons. $D B P$ diastolic
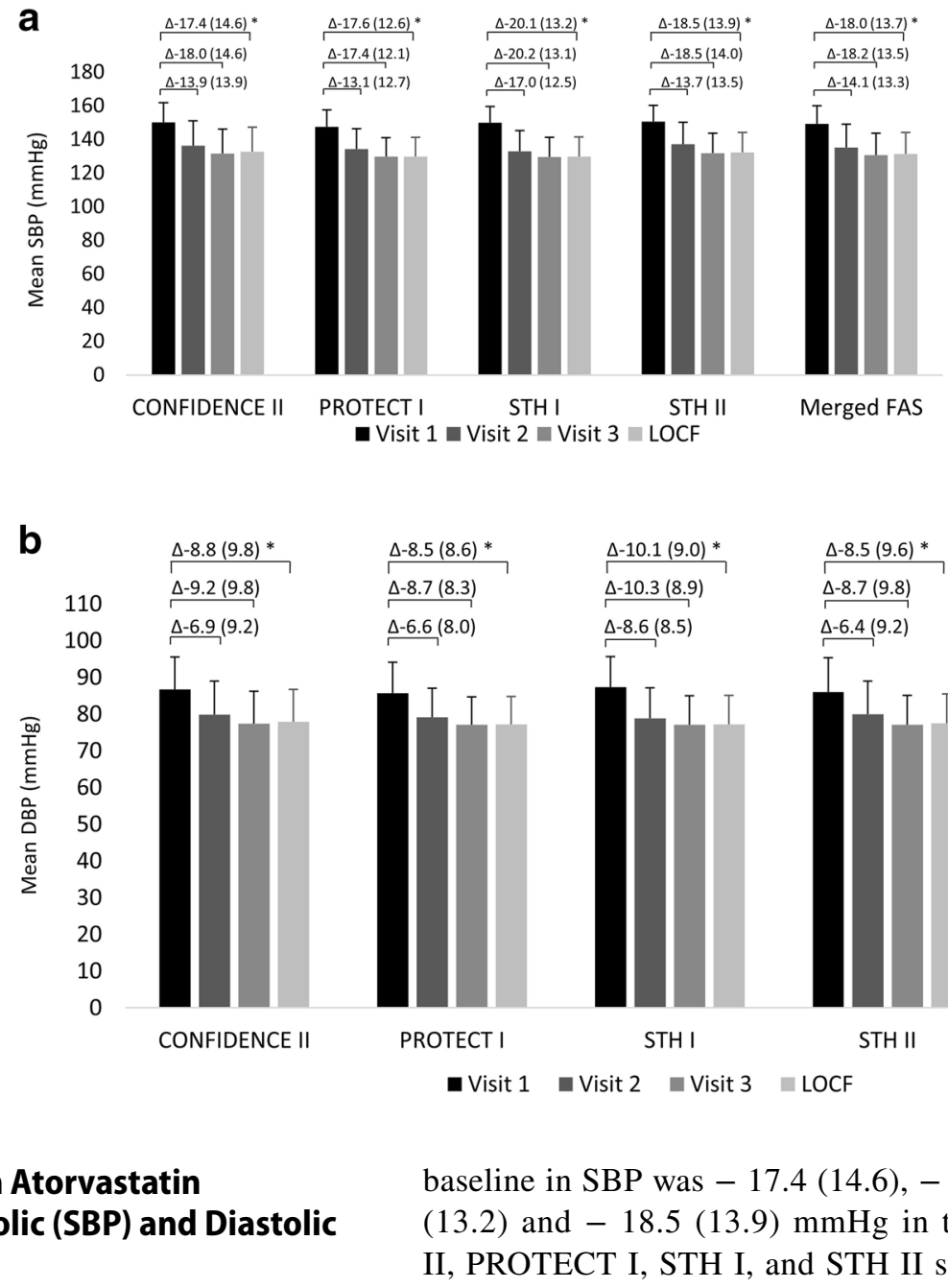

Figure $2 \mathrm{a}, \mathrm{b}$ present the changes from baseline to Visit 2, Visit 3, and the LOCF in SBP and DBP, respectively, for the merged FAS and by individual study. Overall, a timedependent decrease in both SBP and DBP was observed. By individual study, mean (SD) SBP change from baseline was highest in STH I (Visit 2: - 17.0 [12.5] mmHg; Visit 3: -20.2 [13.1] $\mathrm{mmHg}$ ), followed by comparable decreases in CONFIDENCE II, PROTECT I, and STH II at both Visit 2 (- 13.9 [13.9]; - 13.1 [12.7]; - 13.7 [13.5] mmHg, respectively) and Visit 3 (- 18.0 [14.6]; - 17.4 [12.1]; - 18.5 [14.0] $\mathrm{mmHg}$, respectively). Corresponding mean (SD) decreases in DBP observed at Visit 2 ranged from a minimum of -6.4 (9.2) $\mathrm{mmHg}$ (STH II) to a maximum -8.6 (8.5) $\mathrm{mmHg}$ (STH I). At Visit 3, mean (SD) changes in DBP from baseline were - 9.2 (9.8) $\mathrm{mmHg}$ (CONFIDENCE II), - 8.7 (8.3) mmHg (PROTECT I), - 10.3 (8.9) mmHg (STH I), and -8.7 (9.8) $\mathrm{mmHg}$ (STH II).

With respect to the LOCF analysis, significant $(p<0.001)$ within-group changes in SBP and DBP from baseline were observed at LOCF in the merged FAS, as well as within each individual study population: mean (SD) change from baseline in SBP was - 17.4 (14.6), - 17.6 (12.6), - 20.1 (13.2) and -18.5 (13.9) $\mathrm{mmHg}$ in the CONFIDENCE II, PROTECT I, STH I, and STH II studies, respectively (Fig. 2a), with a resultant change in the merged FAS of - 18.0 (13.7) $\mathrm{mmHg}$. The mean (SD) change in DBP for LOCF was -8.8 (9.8) $\mathrm{mmHg}$ in CONFIDENCE II, -8.5 (8.6) $\mathrm{mmHg}$ in PROTECT I, -10.1 (9.0) $\mathrm{mmHg}$ in STH I, -8.5 (9.6) $\mathrm{mmHg}$ in STH II, and -8.9 (9.3) $\mathrm{mmHg}$ in the merged FAS (Fig. 2b).

Patients included in the assessment of target BP at each visit were those with available data for SBP, DBP, and diabetes status. Between 50.1\% $(n=173 / 345$; STH II $)$ and $65.6 \%(n=328 / 500$; STH I) of patients achieved an SBP/ DBP of $<140 / 90 \mathrm{mmHg}$ (or $<140 / 85 \mathrm{mmHg}$ for diabetic patients) by Visit 2 (Table 5). This proportion increased across all groups at Visit 3, to 71.5\% in CONFIDENCE II $(n=702 / 982), 80.0 \%$ in PROTECT I $(n=592 / 740), 80.1 \%$ in STH I $(n=347 / 433)$, and $71.9 \%$ in STH II $(n=207 / 288)$. LOCF analyses resulted in lower although comparable proportions of patients achieving target, which was reported by $68.1 \%$ of patients in CONFIDENCE II $(n=802 / 1177)$, $78.1 \%$ of patients in PROTECT I $(n=676 / 866), 77.4 \%$ of patients in STH I $(n=408 / 527)$, and $70.7 \%$ of patients in STH II $(n=256 / 362)$. This trend was also apparent in the merged FAS, with $56.9 \%(n=1640 / 2880), 75.6 \%$ 
Table 5 By-visit achievement of target blood pressure ${ }^{\mathrm{a}}$ by study and for the merged full analysis set

\begin{tabular}{llllll}
\hline & $\begin{array}{l}\text { CONFIDENCE II } \\
(N=1182)\end{array}$ & $\begin{array}{l}\text { PROTECT I } \\
(N=866)\end{array}$ & $\begin{array}{l}\text { STH I } \\
(N=528)\end{array}$ & $\begin{array}{l}\text { STH II } \\
(N=362)\end{array}$ & $\begin{array}{l}\text { Merged FAS } \\
(N=2938)\end{array}$ \\
\hline $\begin{array}{l}\text { Achievement of tar- } \\
\text { get BP, yes, } n(\%)^{\mathrm{b}}\end{array}$ & & & & & \\
Visit 2 & $N=1171$ & $N=864$ & $N=500$ & $N=345$ & $N=2880$ \\
& $635(54.2)$ & $504(58.3)$ & $328(65.6)$ & $173(50.1)$ & $1640(56.9)$ \\
Visit 3 & $N=982$ & $N=740$ & $N=433$ & $N=288$ & $N=2443$ \\
& $702(71.5)$ & $592(80.0)$ & $347(80.1)$ & $207(71.9)$ & $1848(75.6)$ \\
LOCF & $N=1177$ & $N=866$ & $N=527$ & $N=362$ & $N=2932$ \\
& $802(68.1)$ & $676(78.1)$ & $408(77.4)$ & $256(70.7)$ & $2142(73.1)$ \\
\hline
\end{tabular}

a234 diabetic patients (CONFIDENCE I: $n=71$, PROTECT I: $n=122$, STH I: $n=24$, STH II: $n=17$ ) were considered to have controlled BP at Visit 1, according to the European Society of Hypertension/European Society of Cardiology (ESH/ESC) guidelines, and were excluded from consideration in the target BP analyses

${ }^{\mathrm{b}}$ Target BP was defined as $<140 / 90 \mathrm{mmHg}$ for non-diabetic patients and $<140 / 85 \mathrm{mmHg}$ for diabetic patients; calculated based on the total number of patients with available information (SBP, DBP and diabetic status)

$B P$ blood pressure, $F A S$ full analysis set, $L O C F$ last observation carried forward, STH Shake the Habit
( $n=1848 / 2443)$, and $73.1 \%(n=2142 / 2932)$ of patients achieving target BP at Visit 2, Visit 3, and the LOCF, respectively.

\subsection{Safety}

Of the 3541 patients in the merged safety population, $8.0 \%$ $(n=282 / 3541)$ reported at least one EAE. By individual study, rates were comparable, with $7.4 \%(n=99 / 1332)$ of patients in CONFIDENCE II, 7.8\% $(n=83 / 1069)$ of patients in PROTECT I, 8.2\% $(n=58 / 709)$ of patients in STH I, and 9.7\% $(n=42 / 431)$ of patients in STH II, reporting an EAE (Table 6). The most common EAEs reported were cough $(n=158 / 3541 ; 4.5 \%)$, headache $(n=31 / 3541 ; 0.9 \%)$, dizziness $(n=29 / 3541 ; 0.8 \%)$, fatigue $(n=14 / 3541 ; 0.4 \%)$, and nausea $(n=11 / 3541 ; 0.3 \%)$. No unexpected adverse events were reported (Table 6).

Only one ESAE (angioedema) was reported by one patient in CONFIDENCE II, while three ESAEs (one case of brain neoplasm and two cases of lung neoplasm) were reported by two patients in STH I, with no relation to the observed treatments as evaluated by the physician. No serious adverse events was reported among patients in PROTECT I or STH II.

\section{Discussion}

This post hoc analysis identified 3541 patients, or $8.1 \%$ of the total pooled ITT population, treated with free combination perindopril + atorvastatin in a real-world setting. With respect to BP, significant reductions in both SBP and DBP were observed within the individual studies, as well as in the merged FAS population, decreasing by $-18.0 /-8.9 \mathrm{mmHg}$ for the LOCF. This was despite high rates of $\mathrm{CV}$ risk factors such as physical inactivity (54.3\%), obesity (38.8\%), and diabetes (45.6\%). These results are in line with those previously reported for a perindopril fixed dose combination (FDC)
Table 6 Emergent adverse events by study and for the merged safety population

\begin{tabular}{lccccc}
\hline & $\begin{array}{l}\text { CONFIDENCE II } \\
(N=1332)\end{array}$ & $\begin{array}{l}\text { PROTECT I } \\
(N=1069)\end{array}$ & $\begin{array}{l}\text { STH I } \\
(N=709)\end{array}$ & $\begin{array}{l}\text { STH II } \\
(N=431)\end{array}$ & $\begin{array}{l}\text { Merged safety } \\
(N=3541)\end{array}$ \\
\hline $\begin{array}{l}\text { Total number of EAEs reported } \\
\begin{array}{l}\text { No. of patients reporting at } \\
\text { least one EAE, } n(\%)\end{array}\end{array}$ & 147 & 106 & 73 & 62 & 388 \\
$\begin{array}{l}\text { No. of patients reporting most } \\
\text { common EAE (PT), } n(\%)\end{array}$ & & $83(7.8)$ & $58(8.2)$ & $42(9.7)$ & $282(8.0)$ \\
Cough & $65(4.9)$ & & & & \\
Dizziness & $6(0.5)$ & $46(4.3)$ & $35(4.9)$ & $12(2.8)$ & $158(4.5)$ \\
Fatigue & $7(0.5)$ & $10(0.9)$ & $3(0.4)$ & $10(2.3)$ & $29(0.8)$ \\
Headache & $8(0.6)$ & $14(1.3)$ & $7(1.0)$ & $2(0.5)$ & $31(0.9)$ \\
Nausea & $4(0.3)$ & $2(0.2)$ & $1(0.1)$ & $4(0.9)$ & $11(0.3)$ \\
\hline
\end{tabular}

$E A E$ emergent adverse event $P T$ Preferred term, $S T H$ Shake the Habit 
assessed in routine care: the EMERALD study [21] assessed the effectiveness of combination perindopril/amlodipine in reducing SBP and DBP in 2269 patients with diagnosed essential hypertension. Reductions in SBP/DBP from baseline to week 16 were found to be dependent on baseline BP levels; in Grade I hypertensive patients (defined as baseline SBP $140-159 \mathrm{mmHg}$ or DBP $90-99 \mathrm{mmHg}$ ), for which the merged FAS population presented herein most closely resembles, SBP/DBP reduction to week 16 was found to be $-21.9 /-10.0 \mathrm{mmHg}$. Although the EMERALD study reported a higher proportion of patients achieving target BP ( $88.5 \%$ vs $73.1 \%$ in the current study), the rate of diabetes reported is $20 \%$ higher in the current post hoc analysis and may have contributed to the lower rates of target BP achievement observed. In addition to the significant reductions in BP observed, free combination perindopril + atorvastatin was found to be safe and well tolerated, demonstrating a safety profile similar to that of atorvastatin and perindopril administered as single agents $[22,23]$. The results of this study may also be generalized to the $5-10 \mathrm{mg} /$ day arginine salt formulation of perindopril, which corresponds to the perindopril-tert-butylamine $4-8 \mathrm{mg}$ /day formulations investigated herein. Developed to improve the stability of perindopril in high heat and high humidity environments, the bioequivalence in terms of pharmacokinetics, efficacy, safety, and acceptability of the arginine salt formulation to that to that of tert-butylamine salt has been proven in an open-label, randomized, pharmacokinetic study [24].

In our study, approximately $30-60 \%$ of patients were identified to also receive concurrent ASA/antiplatelet, cardiac therapy, and antihypertensive therapy. As more than one antihypertensive agent is often required to achieve BP control [13, 25], this elevated pill burden is an established clinical reality in routine clinical practice.

However, the advent of single-pill combination antihypertensive agents has shown to be effective in reducing pill burden, and accordingly, has been found to increase patient adherence by up to $24 \%$ compared with the administration of free-drug components [26]. In addition, a more recent study demonstrated that adherence in patients receiving a single calcium channel blocker (amlodipine) + atorvastatin combination pill was 2.1 to 2.5 times greater compared with patients receiving amlodipine and atorvastatin doses separately [27].

A major limitation of the current post hoc analysis is related to the cutoff used for the achievement of target BP in diabetics, updated to reflect the $2013 \mathrm{ESH} / \mathrm{ESC}$ recommendation current at the time of the post hoc analysis $(140 / 85 \mathrm{mmHg})$ [19]. As the 2012 CHEP guideline thresholds were implemented at the time of the individual study conduct [20], a discrepancy was noted whereby protocol-defined uncontrolled hypertension in diabetics was established at BPs $\geq 130 / 80 \mathrm{mmHg}$, resulting in 234 diabetic patients considered to have controlled hypertension at baseline (Visit 1) due to post hoc adoption of European standards. Although included in the individual and merged safety and FAS populations, these patients were excluded from the target BP analyses in order to provide a more accurate representation of $\mathrm{BP}$ control.

A second limitation is related to the lack of lipid parameters collected: as the objectives of each individual study were to assess the effectiveness of perindopril in controlling BP, this post hoc study design, which encompassed a select group of patients with concomitant atorvastatin treatment, was limited to the data collected under the original study objectives. As such, although the effectiveness of perindopril was established in the results presented, whether a simultaneous reduction in cholesterol levels was achieved under the administration of atorvastatin could not be assessed from the current data. This is also true with respect to the lack of data related to patient adherence to both perindopril and atorvastatin, as well as the indication to receive atorvastatin. In addition, patients across all four studies were followed for approximately 16 weeks; although this may not have allowed adequate time for long-term safety evaluation, no unexpected adverse events were reported within this time frame, supporting a need for more lengthy investigations into the combination of perindopril and atorvastatin for treatment of hypertension.

A final limitation of the current post hoc analysis is related to the observational nature of the individual studies included; more specifically, the lack of a comparator arm and randomization. However, the results of this study confer a high degree of generalizability to real-life clinical practice settings, providing important information with respect to hypertensive patient characteristics, and physician practice patterns alike.

Despite these limitations, to our knowledge, this is the first study to investigate perindopril co-administered with a cholesterol-lowering agent for the treatment of mild-tomoderate hypertension. The results of this post hoc analysis have demonstrated that not only is perindopril + atorvastatin co-prescription common in routine clinical practice, but it is also a safe and effective choice for the treatment of patients with mild-to-moderate hypertension and concomitant hypercholesterinemia. Further investigation is warranted to establish the long-term safety and effectiveness of perindopril + atorvastatin in a real-world setting, to describe the rate of adherence, as well as explore effects on lipid parameters. In addition, the results of this study may also be used to inform early phase trials assessing the efficacy and safety of perindopril + atorvastatin free-drug combination regimens, or of their FDC counterparts, for the treatment of mild-tomoderate hypertension and concomitant hyperlipidemia. 


\section{Compliance with Ethical Standards}

This study was funded by l'Institut de Recherches Internationales Servier (IRIS). JSS, EP, MS, JR, and ER were employees of JSS Medical Research Inc. Each of the four studies included in this post hoc analysis were approved by an Independent Review Board, with individual patient consent obtained.

Open Access This article is distributed under the terms of the Creative Commons Attribution-NonCommercial 4.0 International License (http://creativecommons.org/licenses/by-nc/4.0/), which permits any noncommercial use, distribution, and reproduction in any medium, provided you give appropriate credit to the original author(s) and the source, provide a link to the Creative Commons license, and indicate if changes were made.

\section{References}

1. Lim SS, Vos T, Flaxman AD, Danaei G, Shibuya K, Adair-Rohani $\mathrm{H}$, et al. A comparative risk assessment of burden of disease and injury attributable to 67 risk factors and risk factor clusters in 21 regions, 1990-2010: a systematic analysis for the Global Burden of Disease Study 2010. Lancet. 2012;380(9859):2224-60.

2. Sawicka K, Szczyrek M, Jastrzębska I, Prasał M, Zwolak A, Daniluk J. Hypertension-the silent killer. J Pre-Clin Clin Res. 2011;5(2):43-6.

3. Dalal JJ, Padmanabhan TN, Jain P, Patil S, Vasnawala H, Gulati A. LIPITENSION: interplay between dyslipidemia and hypertension. Indian J Endocrinol Metab. 2012;16(2):240-5.

4. Castelli WP, Anderson K. A population at risk. Prevalence of high cholesterol levels in hypertensive patients in the Framingham Study. Am J Med. 1986;80(2A):23-32.

5. Stamler J, Wentworth D, Neaton JD. Prevalence and prognostic significance of hypercholesterolemia in men with hypertension. Prospective data on the primary screenees of the Multiple Risk Factor Intervention Trial. Am J Med. 1986;80(2A):33-9.

6. Silva PM, Cardoso SM, Ferreira AM. Persistent lipid abnormalities in patients with hypertension and dyslipidemia treated with statins: results of the Portuguese hypertensive subpopulation of the Dyslipidemia International Study (DYSIS). Clin Exp Hypertens. 2015;37(2):116-21.

7. Leiter LA, Lundman P, da Silva PM, Drexel H, Junger C, Gitt AK, et al. Persistent lipid abnormalities in statin-treated patients with diabetes mellitus in Europe and Canada: results of the Dyslipidaemia International Study. Diabet Med. 2011;28(11):1343-51.

8. Jameson K, Amber V, D'Oca K, Mills D, Giles A, Ambegaonkar B. Impact of lipid-lowering therapy on the prevalence of dyslipidaemia in patients at high-risk of cardiovascular events in UK primary care - a retrospective database study. Int J Clin Pract. 2013;67(12):1228-37.

9. Erdine S, Redon J, Bohm M, Ferri C, Kolloch R, Kreutz R, et al. Are physicians underestimating the challenges of hypertension management? Results from the Supporting Hypertension Awareness and Research Europe-wide (SHARE) survey. Eur J Prev Cardiol. 2013;20(5):786-92.

10. Feldman RD, Liu L, Wu Z, Zhang Y, Yu X, Zhang XH. Hypertension Attitude PersPEctives and Needs (HAPPEN): a real-world survey of physicians and patients with hypertension in China. J Clin Hypertens (Greenwich). 2017;19(3):256-64.

11. Redon J, Erdine S, Bohm M, Ferri C, Kolloch R, Kreutz R, et al. Physician attitudes to blood pressure control: findings from the Supporting Hypertension Awareness and Research Europe-wide survey. J Hypertens. 2011;29(8):1633-40.
12. Wong ND, Lopez VA, L'Italien G, Chen R, Kline SE, Franklin SS. Inadequate control of hypertension in US adults with cardiovascular disease comorbidities in 2003-2004. Arch Intern Med. 2007;167(22):2431-6.

13. Writing Group M, Mozaffarian D, Benjamin EJ, Go AS, Arnett DK, Blaha MJ, et al. Heart Disease and stroke statistics-2016 update: a report from the American Heart Association. Circulation. 2016;133(4):e38-360.

14. Ho PM, Magid DJ, Shetterly SM, Olson KL, Maddox TM, Peterson PN, et al. Medication nonadherence is associated with a broad range of adverse outcomes in patients with coronary artery disease. Am Heart J. 2008;155(4):772-9.

15. Jain S, Birbager DR, Boyrazian HA, Sampalis JS, for the PROTECT II/SHAKE THE HABIT I Investigators. Effectiveness of Patient Programs Designed to Increase Physical Activity or Decrease Salt Consumption on Blood Pressure Control. Poster presentation at the $2014 \mathrm{CHC}$ annual conference, Gatineau, Canada; October 16-18, 2014.

16. Jain S, Birbrager DR, Kumar N, Sampalis J, for the SHAKE THE HABIT I Investigators. Effectiveness of a patient support program designed to facilitate salt intake reduction in patients with mild to moderate hypertension treated with perindopril (Shake the Habit I). Oral presentation at the 2014 joint meeting of the ESH-ISH, Athens, Greece; June 13-16, 2014.

17. Jain S, Boucher D, Chen JSL, Sampalis S for the PROTECT I Investigators. Effectiveness of PeRindOpril in the management of hyperTension: idEntification of patient and physiCian determinants of response to Treatment: Findings from PROTECT trial. Poster presentation at the 2014 joint meeting of the ESH-ISH, Athens, Greece; June 13-16, 2014.

18. Vasilevski M, Hamel R, Rampakakis E, Sampalis JS. Impact of Stress Reduction on Blood Pressure Control in Patients Treated with Perindopril; Shake the Habit II. Poster presentation at the European Society of Hypertension Conference, June 10-13, 2016. Paris, France.

19. Task Force M, Montalescot G, Sechtem U, Achenbach S, Andreotti F, Arden C, et al. 2013 ESC guidelines on the management of stable coronary artery disease: the Task Force on the management of stable coronary artery disease of the European Society of Cardiology. Eur Heart J. 2013;34(38):2949-3003.

20. Daskalopoulou SS, Khan NA, Quinn RR, Ruzicka M, McKay DW, Hackam DG, et al. The 2012 Canadian hypertension education program recommendations for the management of hypertension: blood pressure measurement, diagnosis, assessment of risk, and therapy. Can J Cardiol. 2012;28(3):270-87.

21. Vlachopoulos C, Grammatikou V, Kallistratos M, Karagiannis A. Effectiveness of perindopril/amlodipine fixed dose combination in everyday clinical practice: results from the EMERALD study. Curr Med Res Opin. 2016;32(9):1605-10.

22. Black DM, Bakker-Arkema RG, Nawrocki JW. An overview of the clinical safety profile of atorvastatin (lipitor), a new HMG-CoA reductase inhibitor. Arch Intern Med. 1998;158(6):577-84.

23. Clark LT. Safety profile of perindopril. Am J Cardiol. 2001;88(7A):36i-40i.

24. Telejko E. Perindopril arginine: benefits of a new salt of the ACE inhibitor perindopril. Curr Med Res Opin. 2007;23(5):953-60.

25. Piepoli MF, Hoes AW, Agewall S, Albus C, Brotons C, Catapano AL, et al. 2016 European Guidelines on cardiovascular disease prevention in clinical practice: the Sixth Joint Task Force of the European Society of Cardiology and Other Societies on Cardiovascular Disease Prevention in Clinical Practice (constituted by representatives of 10 societies and by invited experts)Developed with the special contribution of the European Association for Cardiovascular Prevention \& Rehabilitation (EACPR). Eur Heart J. 2016;37(29):2315-81. 
26. Bangalore S, Kamalakkannan G, Parkar S, Messerli FH. Fixeddose combinations improve medication compliance: a meta-analysis. Am J Med. 2007;120(8):713-9.

27. Hussein MA, Chapman RH, Benner JS, Tang SS, Solomon HA, Joyce A, et al. Does a single-pill antihypertensive/lipid-lowering regimen improve adherence in US managed care enrolees? A nonrandomized, observational, retrospective study. Am J Cardiovasc Drugs. 2010;10(3):193-202. 